REVISTA CIENCIAS BIOMÉ DICAS

PRESENTACIÓN DE CASOS CLÍNICOS

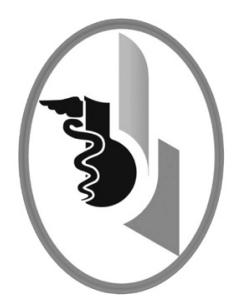

\title{
FUSARIOSIS DISEMINADA POR FUSARIUM VERTICILLIOIDES EN UN PACIENTE CON LEUCEMIA MIELOIDE AGUDA
}

\author{
DISSEMINATED FUSARIUM VERTICILLIOIDES IN A PATIENT \\ WITH ACUTE MYELOID LEUKEMIA
}

\author{
Lobatón-Ramírez José ${ }^{1}$ \\ Coronado-Negrete Auxiliadora ${ }^{2}$ \\ Ramírez-Barranco Rosangela ${ }^{3}$ \\ Rocha-Reyes Ernesto ${ }^{4}$ \\ Pinto-Angarita Jean Carlos ${ }^{4}$ \\ Borré-Naranjo Diana ${ }^{5}$
}

Correspondencia: rosangelaramirezb@gmail.com.

Recibido para evaluación: marzo-21-2016. Aceptado para publicación: octubre-25-2016

\section{RESUMEN}

Introducción: las micosis invasivas son causa de morbilidad y mortalidad en pacientes inmunocomprometidos. De las micosis invasivas causadas por hongos filamentosos, las más frecuentes son la infección por Aspergillus seguida de la producida por las hialohifomicosis del género Fusarium. Este hongo habita en restos orgánicos, suelo y abastecimientos de agua hospitalarios. La vía respiratoria es el principal sitio de entrada, seguido de la piel. Su presentación clínica es inespecífica, por ello la infección pulmonar es la afección más frecuente. El diagnóstico se realiza mediante cultivo del hongo y es uno de los pocos hongos que crecen en los hemocultivos. El objetivo fue presentar un caso clínico de fusariosis diseminada por Fusarium verticillioides en un paciente afectado con leucemia mieloide aguda.

Caso clínico: describimos un caso de fusariosis pulmonar con compromiso de piel y tejidos blandos en un paciente adolescente con actividad en zona rural, afectado de leucemia mieloide aguda, quien desarrolló el cuadro infeccioso durante la quimioterapia de inducción.

Conclusión: la mortalidad es alrededor del 80\%, por tanto un elevado índice de sospecha, la confirmación del diagnóstico y el tratamiento oportuno son importantes. Rev.cienc.biomed. 2017;7(2):316-322.

\section{PALABRAS CLAVE}

Fusarium; Leucemia; Micosis.

1 Médico. Especialista en Hemato-oncología. Instituto Médico de Alta Tecnología (IMAT). Oncomedica S.A. Montería. Colombia.

2 Microbióloga. Instituto Médico de Alta Tecnología (IMAT). Oncomedica S.A Montería. Colombia.

3 Médico. Especialista en Epidemiología. Estudiante de Posgrado. Medicina Interna. Facultad de Medicina. Universidad de Cartagena. Cartagena. Colombia.

4 Médico. Estudiante de Posgrado. Medicina Interna. Facultad de Medicina. Universidad de Cartagena. Cartagena. Colombia.

5 Médico. Internista. Universidad de Cartagena. Colombia. 
ISSN: 2215-7840, 7(2), julio-diciembre 2016, Lobatón-Ramírez José, Coronado-Negrete Auxiliadora, Ramírez-Barranco Rosangela,

Rocha-Reyes Ernesto, Pinto-Angarita Jean Carlos, Borré-Naranjo Diana

\section{SUMMARY}

Introduction: invasive mycoses are cause of morbidity and mortality in immunocompromised patients. Among invasive mycoses caused by filamentous fungi, the most frequent are Aspergillus infection followed by hyalohyphomycosis of the genus Fusarium. This fungus inhabits organic remains, soil and hospital water supplies. The airway is the main entry site, followed by the skin. Its clinical presentation is non-specific, for that reason, pulmonary infection is the most common condition. The diagnosis is made by cultivating the fungus; it is one of the few fungi that grow in blood cultures. The objective was to present a case report of fusariosis disseminated by Fusarium verticillioides in a patient affected with acute myeloid leukemia.

Case report: to describe a case about pulmonay fusariosis with compromise in the skin and soft tissues in an adolescent patient who lives in a rural zone. The patient was suffering from acute myeloid leukemia and infectious disease during induction chemotherapy.

Conclusion: the mortality is around $80 \%$. Therefore, to confirm the diagnosis and timely treatment are important. Rev.cienc.biomed. 2017;7(2):316-322.

\section{KEYWORDS}

Fusarium; Leukemia; Mycoses.

\section{INTRODUCCIÓN}

En general las micosis más frecuentes son las causadas por hongos del género Candida y Aspergillus, pero existen otros que afectan al paciente que se encuentra con compromiso inmunológico y son llamados emergentes (1). Suelen crecer por factores diversos, especialmente a consecuencia de los esquemas de quimioterapia e inmunosupresores, al uso de antibacterianos y al aumento de la utilización de antifúngicos como terapia profiláctica empírica (2).

Los hongos Fusarium son principalmente patógenos vegetales encontrados en plantas y material inorgánico. La fusariomicosis está incluida dentro de las hialohifomicosis (micosis causadas por hongos filamentosos, tabicados, hialinos) que son la contraposición a la feohifomicosis (causadas por hongos filamentosos negros). Producen infecciones diseminadas e invasivas en huéspedes inmunocomprometidos, se adquiere a través de la inhalación de conidias dispersas en el aire o a través de fisuras en la piel (1).

Los cambios climáticos y el uso de drogas inmunosupresoras predisponen a este tipo de enfermedades micóticas (2). La fusariosis es la segunda causa de infección micótica generada por hongos filamentosos, después de la aspergilosis, afectando principalmente a pacientes con enfermedad neoplásica hematológica, en quimioterapia o posterior a trasplante hematopoyético (3).

Las manifestaciones de las infecciones por Fusarium pueden ser desde superficiales con compromiso cutáneo, hasta invasión local y posterior forma diseminada. El diagnóstico se realizará con estudio histopatológico, tinción de Gram, hemocultivos o serología (4). El pronóstico es reservado ya que la mortalidad de la forma diseminada es alrededor del $80 \%$ (5). En el tratamiento se emplea anfotericina B, voriconazol, posaconazole 0 isavuconazole. Puede ser importante el desbridamiento de las lesiones (6). El objetivo es presentar un caso clínico de fusariosis diseminada por Fusarium verticillioides en un paciente afectado con leucemia mieloide aguda.

\section{CASO CLÍNICO}

Paciente masculino de 19 años de edad, residente en área rural del departamento de Córdoba, Colombia, en contacto con cultivos de maíz, quien ingresó al Instituto Médico de Alta Tecnología IMAT Oncomédica S.A. Montería, Córdoba, Colombia en mayo de 2016 con cuadro clínico de tres semanas de evolución de fiebre no cuantificada asociada a astenia y adinamia.

A su ingreso el paciente estaba afebril, taquicárdico, con palidez mucocutánea 
generalizada, sin adenopatías o megalias, alteración neurológica o lesiones en piel. En el hemograma se encontró hemoglobina de $7 \mathrm{~g} / \mathrm{dL}$, trombocitopenia moderada con plaquetas en $55 \times 10^{3} / \mathrm{uL}$ y leucocitosis con $59.9 \times 10^{3} / \mathrm{uL}$. Presencia de blastos en sangre periférica y neutrófilos absolutos de $0.59 \times 10^{3} /$ uL. Se realizó aspirado, biopsia y citometría de flujo de biopsia de médula ósea identificándose presencia de leucemia mieloide aguda. Se inició quimioterapia de inducción con esquema 7+3 (citarabina 100 $\mathrm{mg} / \mathrm{SC}$ por siete días y daunorrubicina 45 $\mathrm{mg} / \mathrm{SC}$ por tres días).

Al noveno día de quimioterapia el paciente presentó temperatura de $40^{\circ} \mathrm{C}$ asociada a neutropenia profunda, neutrófilos absolutos $0.016 \times 10^{3} / \mathrm{uL}$, por lo que se inició manejo empírico para neutropenia febril con cefepime y a las 72 horas, ante la presencia de mucositis grado IV, se adicionó vancomicina. No se observó respuesta favorable hematológica y por la presencia de neutropenia prolongada, se practicó cubrimiento para hongos con caspofungina. Se presentaron en ese momento en las extremidades y en la cara, lesiones eritematosas de centro necrótico sugestivas de ectima gangrenoso (Figura $N^{\circ} 1$ ). También se presentaron nódulos subcutáneos dolorosos en extremidades inferiores y tórax. En valoración ultrasonográfica se consideró a las lesiones como ecomixtas y de origen inflamatorio.

Los hemocultivos iniciales fueron negativos a las 72 horas. Ante la presencia de rinorrea y congestión nasal se indicó tomografía computarizada de senos paranasales y tórax, reportándose pansinusitis, obstrucción del ostium y presencia de lesiones nodulares irregulares con centro necrótico a nivel del lóbulo inferior derecho y del lóbulo medio compatible con neumonía nodular. Figura No 2.

Se realizó nasofibroscopía para tomar muestra para cultivo de secreción de la fosa nasal. Fusarium verticillioides fue aislado en esta secreción a las 96 horas (Figura No 3). También se observaron en hemocultivos de control tomados el día séptimo tras el inicio de la neutropenia febril. Figura No 4.
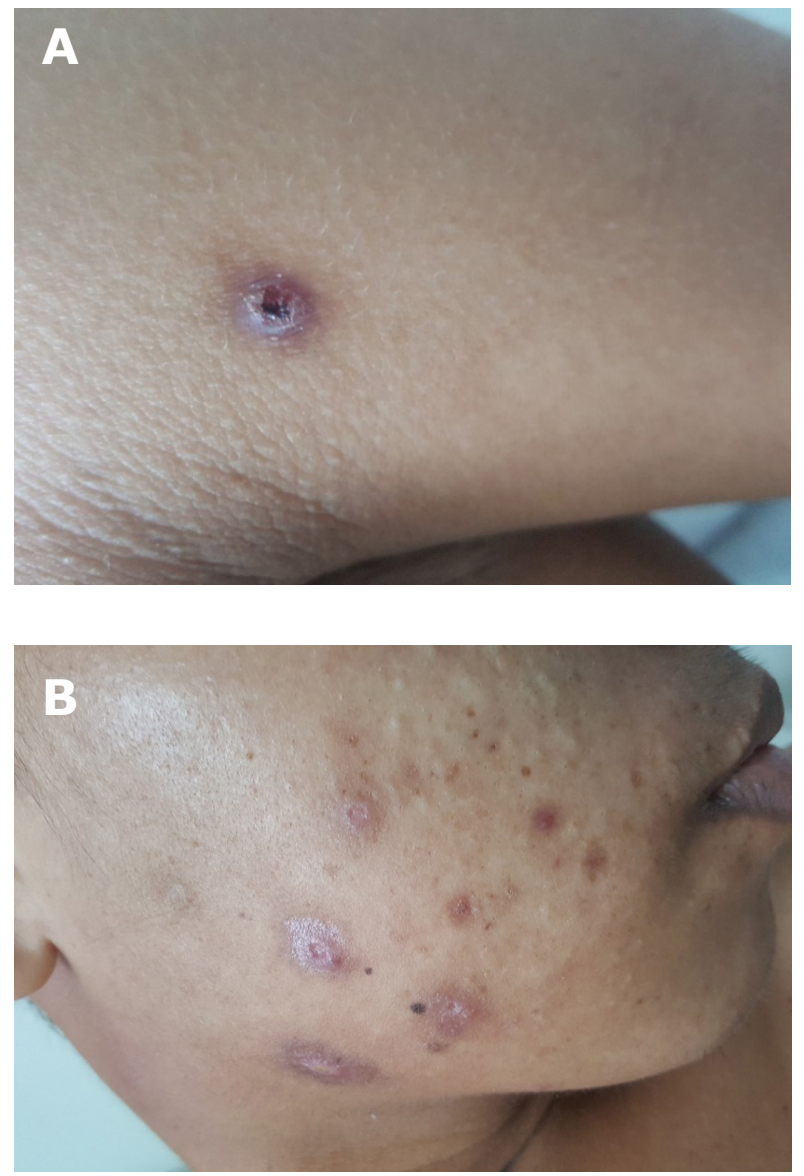

FIGURA NO 1.

(A). Lesiones iniciales de piel.

(B). Luego de cinco días de tratamiento con antifúngico.

Se suspendieron los antimicrobianos y se modificó el esquema antifúngico. Se ordenó anfotericina B a dosis de $5 \mathrm{mg} / \mathrm{kg} /$ día inicial, seguido de $3 \mathrm{mg} / \mathrm{kg} /$ día. También voriconazol a $6 \mathrm{mg} / \mathrm{kg} /$ día inicial y seguido de $4 \mathrm{mg} / \mathrm{kg}$ cada 12 horas por dos semanas. El paciente evolucionó de forma satisfactoria de la neutropenia y del cuadro de fusariosis y posteriormente ordenada de alta para seguimiento especializado.

\section{DISCUSIÓN}

Se presenta el caso de un paciente con infección por Fusarium verticillioides, desencadenada por la presencia de neutropenia profunda inducida por la quimioterapia de inducción ante el diagnóstico de leucemia mieloide aguda. Los hongos del género Fusarium son principalmente patógenos vegetales que producen infecciones diseminadas 


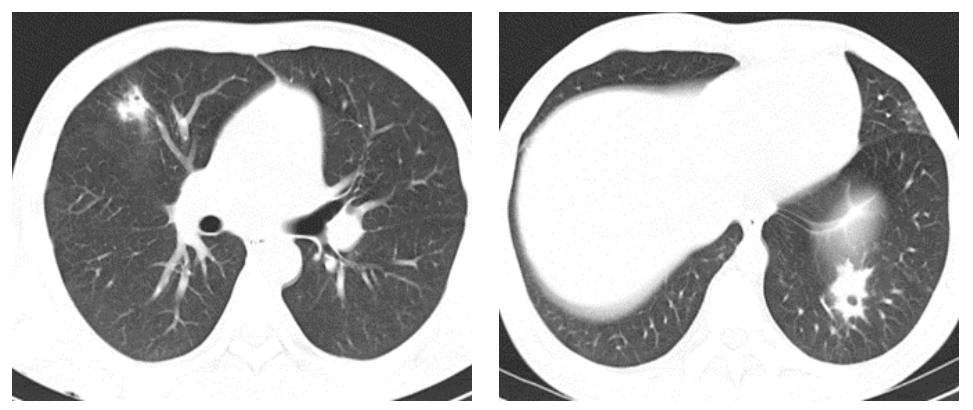

Figura $N^{\circ}$ 2. Tomografía de Tórax. Masa pulmonar cavitada de 31 $\mathrm{mm}$ de contornos irregulares en el lóbulo inferior izquierdo. Lesión nodular de contornos lobulados y bien definidos de $23 \mathrm{~mm}$ en el lóbulo medio. Opacidad en vidrio esmerilado en ambos campos pulmonares. Neumonía nodular.

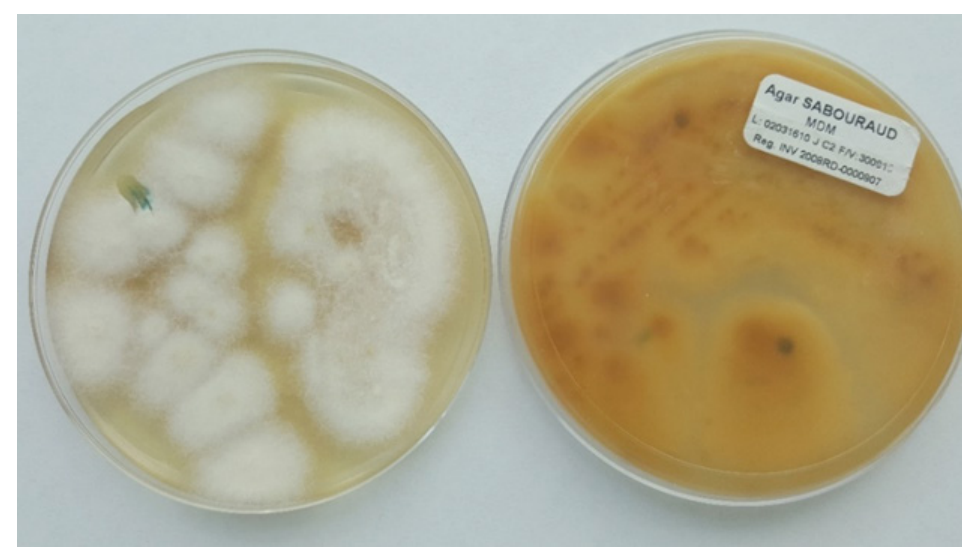

Figura No 3. Cultivo agar sabouraud. Crecimiento de Fusarium verticillioides. Crecimiento micelial abundante, de aspecto algodonoso, con reverso amarillo ocre.
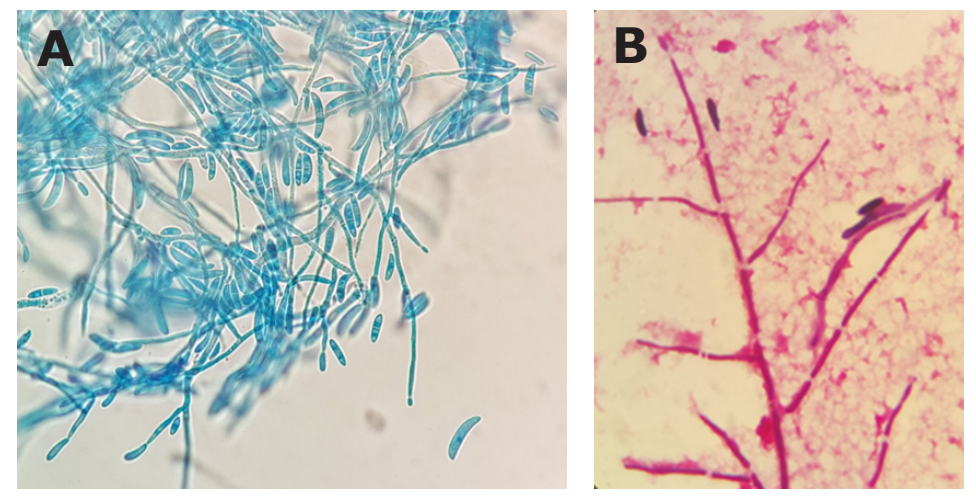

Figura No 4. Micromorfología de Fusarium verticillioides.

(A). Preparación con azul de lactofenol. Se observan hifas delgadas septadas con conidias fusiformes casi rectas $y$, superficie dorsal $y$ ventral paralelas con dos o tres tabiques; conidióforos con monofiálides, laterales sin ramificación; microconidias ovales y ausencia de clamidosporas. Las macroconidias tienen forma de media luna en el cultivo.

(B). Hemocultivo coloreado con tinción de gram; se observan hifas $y$ conidias. en personas con inmunocompromiso severo (1). Después de la aspergilosis, la fusariosis diseminada es la causa más común de infección invasora por hongos filamentosos en pacientes con enfermedades hematológicas malignas o en receptores de trasplantes de progenitores hematopoyéticos. Nucci et al., (7) en un estudio que incluía a 84 personas con neoplasias hematológicas, identificó fusariosis invasiva en el $56 \%$ de los pacientes con leucemia aguda y la mayoría ( $83 \%$ ) presentaban neutropenia profunda y prolongada, de la misma manera que la presentada en el paciente.

Esta micosis tiene una distribución amplia a nivel mundial, con predominio en clima frío y áreas tropicales. El Fusarium verticillioides tiene implicaciones en la economía mundial debido a su capacidad patogénica sobre plantas, capaz de afectar a animales y humanos; se reproduce en áreas tropicales y subtropicales, afectando principalmente plantaciones de maíz, banano, arroz, entre otras (8). El mecanismo de adquisición de la infección en ocasiones es desconocido.

Los hongos del genero Fusarium pueden infectar al hombre a través de la inhalación de micropartículas provenientes del ambiente contaminado. En el caso presentado se identificó la exposición a cultivos de maíz en donde habita el Fusarium verticillioides (9). Otras formas menos frecuentes de contaminación se presentan a través del contacto directo con estructuras cutáneas lesionadas, la contaminación intrahospitalaria de los depósitos de agua y obras de construcción cercanas, a partir de los cuales puede ocurrir la dispersión de las conidias en micropartículas capaces de llegar a las vías respiratorias $(10,11)$.

La presentación clínica depende de la ruta de entrada del hongo, la in- 
tensidad y la duración de la inmunosupresión. A diferencia del huésped inmunocompetente, en donde la onicomicosis y la queratitis son las manifestaciones más frecuentes, la fusariosis diseminada es la forma de presentación más común en los estados de inmunosupresión severa (12). La presentación clínica a menudo incluye fiebre, lesiones cutáneas e infección de senos paranasales y pulmonar, tal como ocurrió en el paciente, en quien la pansinusitis se estableció como la fuente de diseminación del hongo. Los hallazgos cutáneos evolucionaron rápidamente; comúnmente ocurren durante los primeros cinco días en el tronco y en las extremidades. Las lesiones son a menudo múltiples y típicamente se presentan como pápulas eritematosas dolorosas y nódulos violáceos, que cuando se ulceran desarrollan una escara negra o gris; estas se presentan en un $70 \%(2,13)$.

El compromiso de las vías respiratorias bajas es frecuente y los hallazgos radiológicos son específicos en la mayoría de los casos; incluyen infiltrados alveolares e intersticiales, nódulos y lesiones cavitarias (12). La TC de tórax presentó lesiones nodulares que fueron atribuidas a infección fúngica, al ser aislado en las vías respiratorias altas y hemocultivos positivos para Fusarium verticillioides.

En relación a los estudios microbiológicos solo se realizó galactomanano, no se solicitó 1.3 b D-glucano. El test galactomanano y el test 1.3-b-D-glucano son de valor limitado en el diagnóstico de la infección por Fusarium. El galactomanano suele ser negativo (como ocurrió en este paciente), mientras que el 1.3-b-D-glucano puede ser positivo, pero no específico (3). Los hemocultivos no reportaron ningún crecimiento de bacterias gram positivas o gram negativas descartando infección bacteriana.

Dentro de los denominados hongos oportunistas, las especies de género Fusarium se identifican frecuentemente $(50 \%)$ en los hemocultivos y también mediante el estudio histológico de las lesiones cutáneas con un alto rendimiento diagnóstico $(14,15)$. El Fusarium verticillioides antiguamente llamado Fusarium moniliforme es un hongo filamentoso que crece a temperaturas entre $23-37^{\circ} \mathrm{C}$ (promedio $25^{\circ} \mathrm{C}$ ) y forma colonias de color blanco-pálido a salmón y textura polvorienta debido a la producción de microconidias (16).

El diagnóstico se basó en el aislamiento del hongo de sangre en frascos de hemocultivo (Sistema VersaTREK REDOX 1) a $37^{\circ} \mathrm{C}$ y posterior repique (a las 72 horas de incubación, postaviso de alarma) en Agar Sabouraud, y el tomado de secreción nasal, en Agar Sabouraud MDM, ambos incubados a temperatura ambiente $\left(23^{\circ} \mathrm{C}\right)$. En las siguientes 72 horas de incubación se detectó crecimiento en ambas muestras. El tiempo de incubación fue prolongado, solo hasta el día quinto se pudo conseguir crecimiento maduro y formación de estructuras características para la identificación.

En el extendido a las 72 horas de incubación del frasco de hemocultivo, coloreado con tinción de Gram, se observaron estructuras fúngicas alargadas, semejantes a conidias e hifas hinchadas e irregulares. A los ocho días se observaron hifas delgadas ramificadas tabicadas y conidias fusiformes. En el repique del hemocultivo en Agar Sabouraud se manifestó crecimiento micelial abundante, de aspecto algodonoso, con reverso amarillo ocre. La morfología macro y microscópica fueron datos importantes para la identificación, tomando por referencia los parámetros descritos en la literatura $(17,18,19)$ para lo cual se realizaron microcultivos donde se observaron al microscopio, en preparación con azul de lactofenol, hifas delgadas septadas con conidias fusiformes casi rectas $y$, superficie dorsal y ventral paralelas con dos o tres tabiques; conidióforos con monofiálides, laterales sin ramificación; microconidias ovales y ausencia de clamidosporas.

Actualmente, en la identificación de los hongos filamentosos se han utilizado sus características macroscópicas en forma de las colonias y observación microscópica de hifas, esporas y conidias, las cuales siguen siendo limitadas para definir sus especies; la dificultad de los métodos convencionales reside en su uso en cultivos mixtos, los cuales en realidad muchas veces son complejos de especies similares casi indistinguibles. 
ISSN: 2215-7840, 7(2), julio-diciembre 2016, Lobatón-Ramírez José, Coronado-Negrete Auxiliadora, Ramírez-Barranco Rosangela,

Rocha-Reyes Ernesto, Pinto-Angarita Jean Carlos, Borré-Naranjo Diana

Lo ideal es a partir de nuevas técnicas de identificación y caracterización de hongos propuestas mediante espectrofotometría de masa (MALDI-TOF MS, actualmente ofrece incluso la identificación directamente desde los hemocultivos y el sistema VITEK MS que utiliza colonias) (18) y los ensayos de PCR en tiempo real (20).

El más potente antifúngico in vitro es la anfotericina B y el menos eficaz es el itraconazol. Los azoles exhiben concentraciones inhibitorias mínimas inferiores contra las cepas de Fusarium verticillioides. El posaconazol ha demostrado el mejor rendimiento (21). El voriconazol tiene un amplio espectro en el tratamiento de fungiemia invasiva incluyendo aspergilosis, candidiasis y la infección por Fusarium (22). Las tasas de supervivencia con o sin enfermedad diseminada oscilan entre el 33 y el $50 \%$ de los casos respectivamente; las terapias que han demostrado mejor tasa de supervivencia se realizan con posaconazol y voriconazol (23). También se han empleado combinaciones de tratamiento con anfotericina B liposomal y voriconazol, tal como ocurrió en este caso. Hay estudios que reportan la terapia combinada de anfotericina B liposomal, caspofungina o erbinafina con voriconazol, sin ser superior a la monoterapia con voriconazol (24).

\section{CONCLUSIÓN}

La infección invasiva por Fusarium es la segunda causa de infección invasiva por hongos filamentosos después de la aspergilosis. Fusarium afecta principalmente a pacientes con inmunocompromiso, se presenta en cualquier época del año, con más frecuencia en épocas húmedas, cuando el aire y la lluvia favorecen las condiciones para la diseminación de las esporas. En algunos casos es probable que el paciente esté colonizado antes del ingreso hospitalario y que posterior a la administración de esquemas de quimioterapia e inmunosupresores, amplio uso de antibacterianos, desarrollo de neutropenia febril profunda y prolongada, se desarrolle la infección. Tiene una alta tasa de morbimortalidad en el paciente hematológico, aunque se ha logrado mejoría de la supervivencia con el tratamiento con posaconazol o voriconazol.

CONFLICTO DE INTERESES: ninguno que declarar.

FINANCIACIÓN: recursos propios de los autores. Estudios de laboratorio, insumos hospitalarios, medicamentos y honorarios profesionales aportados dentro de la atención asistencial.

\section{REFERENCIAS BIBLIOGRÁFICAS}

1. Olivares R, Alfaro J, Díaz C, Thompson L. Fusariosis diseminada por Fusarium oxysporum en un paciente adulto con leucemia mieloide aguda y neutropenia severa febril. Rev Chil Infect. 2005;22(4):356-60.

2. Kollipara R, Peranteau AJ, Nawas ZY, Tong Y, Woc-Colburn L, et al. Emerging infectious diseases with cutaneous manifestations. J Am Acad Dermatol. 2016; 75(1):19-30.

3. Muhammed M, Coleman JJ, Herman CA, et al. The challenge of managing fusariosis. Virulence. 2011;2:91-6.

4. Duane R. Hospenthal. Mandell, Douglas y Bennett. Enfermedades infecciosas. Principios y práctica. Hongos poco frecuentes y especies relacionadas. 8a Edición. 2016. Elsevier España. Cap. 270, pag. 3175-87.

5. Pereiro M, Gómez S, Rodríguez L, Toribio J. Micosis más frecuente en inmunodeprimidos. Piel (Barc., Ed. Impr.) 2010;25(2):91-9.

6. Delia M, Monno R, Gianelli G, Ianora AA, Dalfino L, Pastore D, et al. Fusariosis in a patiente with acute mieloid leukaemia: a case report and review of the literatura. Mycopathologia. 2016;181(5-6):457-63.

7. Nucci M, Anaissie EJ, Queiroz-Telles F, et al: Outcome predictors of 84 patients with hematologic malignancies and Fusarium infection. Cancer. 2003;98:315-19.

8. Liu SY, Wang NC, Ye RH, Kao YG. Disseminated Fusarium infection in a patient with acute lymphoblastic leukemia: A case report and review of the literatura. Oncology letters 2014;7:334-36.

9. Czembor E, Ste, pien Ò, Was 'kiewicz A. Effect of environmental factors on Fusarium species and associated mycotoxins in maize grain grown in Poland. PLoS One. 2015;10:336-44.

10. García-Ruiz JC, Olazábal I, Pedroso R. Disseminated fusariosis and hematologic malignancies, a still devastating association. Report of three new cases. Rev Iberoam Micol. 2015;32(3):190-96. 
11. Alangaden G. Nosocomial fungal infections: epidemiology, infection control, and prevention. Infect Dis Clin N Am. 2011;25:201-25.

12. Nucci M, Anaissie E. Fusarium infections in immunocompromised patients. Clin Microbiol Rev. 2007;20:(33):695-704.

13. Le Clech L, Hutin P, Le Gal S, Guillerm G. Skin nodule in a patient with acute lymphoblastic leukaemia. BMJ Case Rep. 2014;1-5.

14. Cocchi S, Codeluppi M, Venturelli C Et al. Fusarium verticillioides fungemia in a liver transplantation patient: successful treatment with voriconazol. Diagnostic Microbiology and Infectious Disease. 2011;71:438-41.

15. Campo M, Lewis RE, Kontoyiannis DP. Fusarosis invasiva en pacientes con neoplasias hematológicas en un centro oncológico: 1998-2009. J Infect. 2010;60: 331-7.

16. Esposto MC, Prigitano A, Tortorano AM. Fusarium musae as cause of superficial and deepseated human infections. Journal de Mycologie Médicale. 2016;16:126-33.

17. Martínez-Hernández L, Caro-Sánchez C, Bonifaz A. Infecciones por Fusarium. Dermatol Rev Mexico. 2014;58:432-42.

18. Cantón-Lacasa E, García-Rodríguez J, Guinea-Ortega JV, Martín-Mazuelos E, Pemán J, Guinea J. Métodos microbiológicos para el diagnóstico, manejo y estudio de la infección fúngica invasora: Enferm Infecc Microbiol Clin. 2014;32(6):375-79.

19. Monzón A, Rodríguez Tudela JL. Infecciones causadas por el género Fusarium. Sociedad Española de Enfermedades Infecciosas y Microbiología Clínica. Internet. (Aceso: 27/07/2016). Disponible en https://www.seimc.org/contenidos/ccs/revisionestematicas/micologia/ fusarium.pdf

20. Pasqualini L., Mencacci A., Leli C., Montagna P., Cardaccia A., Cenci E., el al. Diagnóstic performance of a multiple real-time PCR assay in pattients with suspected sepsis hospitalized in an internal medicine ward. J Clin Microbiol. 2012; 50:1285-88.

21. Tortorano AM, Prigitano A, Esposto MC, Arsic Arsenijevic V, Kolarovic J, Ivanovic D, et al. European Confederation of Medical Mycology (ECMM) epidemiological survey on invasive infections due to Fusarium species in Europe. Eur J Clin Microbiol Infect Dis. 2014;33(9):1623-30.

22. Job KM, Olson J, Stockmann C, Constance JE, Enioutina EY, et al. Pharmacodynamic studies of voriconazole: informing the clinical management of invasive fungal infections. Expert Rev Anti Infect Ther. 2016;14(8):731-46.

23. Horn DL, Freifeld AG, Schuster MG, Azie NE, Franks B, Kauffman CA. Treatment and outcomes of invasive fusariosis: review of 65 cases from the PATH Alliance registry. Mycoses. 2014;57(11):652-8.

24. Lortholary O, Obenga G, Biswas $P$ Et al. International retrospective analysis of 73 cases of invasive fusariosis treated with voriconazole. Antimicrob Agents Chemother 2010;54:4446-50.

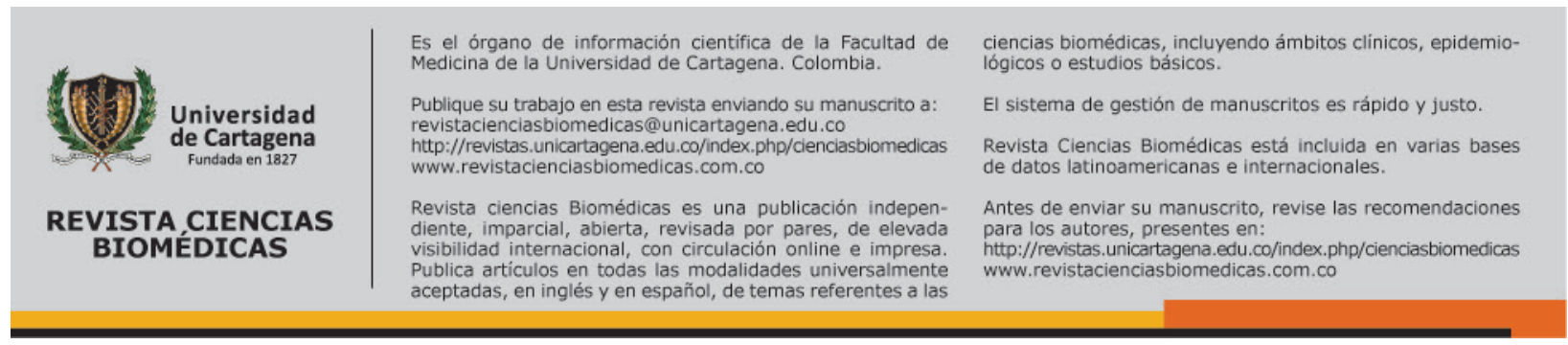

